\title{
INTRINSIC CHIRALITY OF COMPLETE GRAPHS
}

\author{
ERICA FLAPAN AND NIKOLAI WEAVER
}

(Communicated by Frederick R. Cohen)

\begin{abstract}
A graph is said to be intrinsically chiral if no embedding of the graph in 3-space is respected by any ambient orientation-reversing homeomorphism. In this note, we characterize those complete graphs that are intrinsically chiral.
\end{abstract}

It is often important in predicting chemical properties to know whether a molecule is distinct from its mirror image. A molecule that cannot convert itself into its own mirror image is said to be chemically chiral . Mathematically, we consider the molecule as a graph in 3-space and ask whether there is an orientation-reversing homeomorphism that takes the graph to itself. A graph embedded in 3-space is said to be achiral if there exists such a homeomorphism and chiral if there is no such homeomorphism. Note that a molecule whose corresponding graph is chiral is necessarily chemically chiral.

Given that some particular embedding of a graph in 3-space is chiral, it is natural to ask whether it is possible to reembed the graph in such a way that it is now achiral, or whether the chirality is intrinsic to the graph. If there exists some achiral embedding of a graph $G$, we say that $G$ is achirally embeddable; otherwise, we say that $G$ is intrinsically chiral.

The complete graph on $n$ vertices is defined as a set of $n$ vertices together with an edge between each pair of vertices. In this note, we characterize those complete graphs that are intrinsically chiral. In particular, we prove the following

Theorem. The complete graphs $K_{4 n+3}$ with $n \geq 1$ are intrinsically achiral. All other complete graphs $K_{n}$ are achirally embeddable.

First we demonstrate that the complete graphs other than $K_{4 n+3}, n \geq 1$, are achirally embeddable. We do this by actually exhibiting achiral embeddings. The complete graphs $K_{1}, K_{2}$, and $K_{3}$ are planar, and the construction is therefore trivial for them. The next case consists of all graphs $K_{4 n}, n \geq 1$, for which we will give a general form by

Proposition 1. Let $G$ be a finite graph for which there exists an order 4 automorphism $\alpha: G \rightarrow G$ such that the orbit of every vertex under $\alpha$ has length 4 . Then $G$ is achirally embeddable.

Received by the editors January 8, 1990.

1991 Mathematics Subject Classification. Primary 57M25, $05 \mathrm{C} 10$.

Key words and phrases. Embedded graphs, chirality, amphichirality.

(C) 1992 American Mathematical Society $0002-9939 / 92 \$ 1.00+\$ .25$ per page 


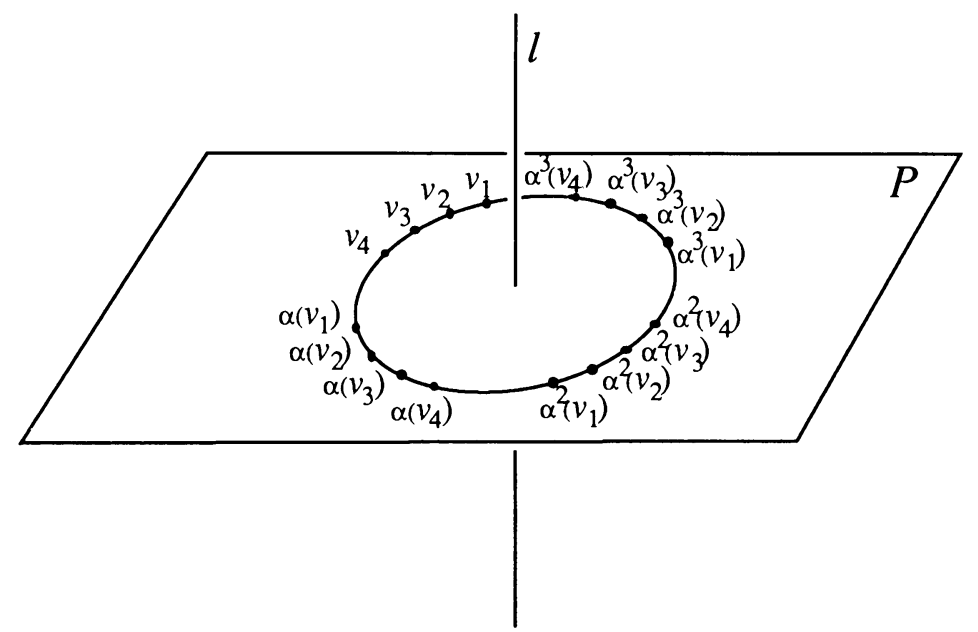

FIGURE 1

Proof. Place the vertices of $G$ on a circle $C$ in a horizontal plane $P \subset \mathbb{R}^{3}$ so that the $90^{\circ}$ rotation about a perpendicular line $l$ induces the same permutation of vertices as $\alpha$ (see Figure 1). Let $f: \mathbb{R}^{3} \rightarrow \mathbb{R}^{3}$ be the composition of the $90^{\circ}$ rotation about $l$ with the reflection through the plane $P$.

Choose $e_{1}, \ldots, e_{p}$ to be representatives from each edge orbit. Then choose ellipsoids $E_{1}, \ldots, E_{p}$ that are symmetric about $l$ and $P$ and meet at the circle $C$ containing the vertices, as in Figure 2. We now embed the edges of $G$ as follows.

The edge $e_{i}$ is invariant under $\alpha^{2}$ if $e_{i}$ joins a vertex $v$ to $\alpha^{2}(v)$. In this case, embed $e_{i}$ in the upper half-ellipsoid $E_{i}^{+}$so that it is invariant under the $180^{\circ}$ rotation $f^{2}$ about $l$. (For instance, take $e_{i}$ to be the intersection of $E_{i}^{+}$ with a vertical plane.) And embed $\alpha\left(e_{i}\right)$ as the image of this edge under $f$, contained in the lower half-ellipsoid $E_{i}^{-}$.

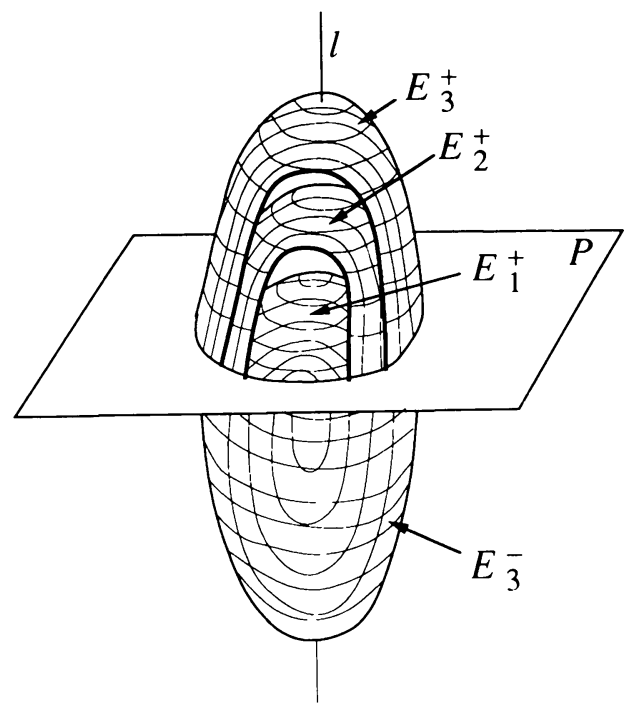

FiguRE 2 
Suppose $e_{i}$ is not invariant under $\alpha^{2}$. Then $e_{i}$ joins a vertex $v$ to a vertex $w$ and $v$ and $w$ are not antipodal points on the circle $C$. Consider the semicircles $A$ and $B$ of $C$ with end points $v$ and $\alpha^{2}(v)$, the antipodal point of $v$. Without loss of generality, $v$ and $w$ are both contained in $A$. Hence, $\alpha^{2}(v)$ and $\alpha^{2}(w)$ are both contained in $B$. Thus an edge joining $v$ to $w$ in the half-ellipsoid $E_{i}^{+}$does not need to meet an edge joining $\alpha^{2}(v)$ to $\alpha^{2}(w)$. So, embed $e_{i}$ in $E_{i}^{+}$so that it is disjoint from its image under $f^{2}$ (again, the intersection of $E_{i}^{+}$with a vertical plane will do). Embed $\alpha\left(e_{i}\right), \alpha^{2}\left(e_{i}\right), \alpha^{3}\left(e_{i}\right)$ as the images of $e_{i}$ under $f, f^{2}, f^{3}$, respectively.

We now have specified an embedding of $G$ in $\mathbb{R}^{3}$, which is invariant under the orientation-reversing homeomorphism $f: \mathbb{R}^{3} \rightarrow \mathbb{R}^{3}$. This proves that $G$ is achirally embeddable.

Corollary. For every $n \geq 1, K_{4 n}$ is achirally embeddable.

Proposition 2. For every $n \geq 1, K_{4 n+1}$ is achirally embeddable.

Proof. First carry out the construction of the proof of Proposition 1 to embed a subgraph $K_{4 n}$ of $K_{4 n+1}$ in $\mathbb{R}^{3}$. Then add the final vertex at the point where the plane $P$ intersects the line $l$, with straight edges connecting this vertex to all the other vertices. The same homeomorphism $f$ now takes the embedded $K_{4 n+1}$ to itself, showing that the embedding is achiral.

Proposition 3. For every $n \geq 1, K_{4 n+2}$ is achirally embeddable.

Proof. Embed a subgraph $K_{4 n}$ of $K_{4 n+2}$ as in the proof of Proposition 1. Let $E_{0}$ be an additional symmetric ellipsoid that is contained in the interior of all the other $E_{i}$. Then add the remaining two vertices at $l \cap E_{0}^{+}$and $l \cap E_{0}^{-}$. Connect these last two vertices to each other by a line segment in $l$ and to the other $4 n$ vertices by intersections of $E_{0}^{ \pm}$with vertical planes. Once again, the homeomorphism $f$ still takes this embedded $K_{4 n+2}$ to itself, showing that the embedding is achiral.

To prove that $K_{4 n+3}$ is intrinsically chiral when $n \geq 1$, we start with a Lemma. Let $f: \mathbb{R}^{3} \rightarrow \mathbb{R}^{3}$ be a homeomorphism that respects an embedding of $K_{7}$ in $\mathbb{R}^{3}$. If the action of $f$ on the vertices of $K_{7}$ has order 2 then $f$ fixes exactly one vertex.

Proof. Our proof is based on a result of Conway and Gordon [CG]. Let a 7-loop in $K_{7}$ be any subgraph that is homeomorphic to a circle and passes through all 7 vertices of $K_{7}$. Given an embedding of $K_{7}$ in $\mathbb{R}^{3}$, such a 7-loop $c$ forms a (possibly trivial) knot in $\mathbb{R}^{3}$, and therefore has a well-defined Arf invariant $a(c) \in \mathbb{Z}_{2}$ (see for instance the appendix of [CG] for the definition of the Arf invariant). Conway and Gordon prove that the $\mathbb{Z}_{2}-\operatorname{sum} \sum_{c \in \mathscr{C}} a(c)$, where $c$ ranges over the set $\mathscr{C}$ of all 7 -loops in $K_{7}$, is always equal to 1 .

Now $f$ acts as an involution on the set $\mathscr{C}$. Since $f$ is a homeomorphism of $\mathbb{R}^{3}, a(f(c))=a(c)$ for every $c \in \mathscr{C}$. Pairing each $c \in \mathscr{C}$ with its image, we conclude that $1=\sum_{c \in \mathscr{C}} a(c)=\sum_{f(c)=c} a(c)$. In particular, $f$ respects some $c_{0} \in \mathscr{C}$. This means that it is possible to choose a 7-tuple of distinct vertices of $K_{7}$ so that $f$ acts on this 7-tuple either by a cyclic permutation or by reversing its order. A cyclic permutation has order 1 or 7. Since the action of $f$ has order 2 on the vertices, it must therefore be an order reversal. It follows that $f$ fixes exactly one vertex of $K_{7}$. 
Proposition 4. For $n \geq 1$, the complete graph $K_{4 n+3}$ is intrinsically chiral.

Proof. Assume that an orientation-reversing homeomorphism $f: \mathbb{R}^{3} \rightarrow \mathbb{R}^{3}$ respects some embedding of $K_{4 n+3}$. Let $n_{1}, \ldots, n_{p}$ denote the lengths of the orbits of the vertices of $K_{4 n+3}$ under the action of $f$. Decompose each $n_{i}$ as $n_{i}=2^{p_{i}} q_{i}$ where $q_{i}$ is odd, and let $q$ be the least common multiple of all the $q_{i}$. Set $g=f^{q}$. Then $g: \mathbb{R}^{3} \rightarrow \mathbb{R}^{3}$ is still orientation-reversing, respects $K_{4 n+3}$, and the lengths of the orbits of the vertices under $g$ are all powers of 2 (including $2^{0}=1$ ).

Since the lengths of the orbits total $4 n+3$ and all orbits have length a power of 2 , there must be at least either three orbits of length 1 or one orbit of length 1 and one orbit of length 2 . In particular, there is a set of 3 vertices of $K_{4 n+3}$ that is invariant under $g$ and fixed pointwise by $g^{2}$.

Suppose that there is at least one orbit of length $2^{m}$ with $m \geq 2$. Then $g^{2^{m-2}}$ has an orbit of length 4 . The union of this orbit with the above $g$-invariant set of 3 vertices now forms a $g^{2^{m-2}}$-invariant set of 7 vertices of $K_{4 n+3}$ and spans a subgraph isomorphic to $K_{7}$. Then $g^{2^{m-1}}=\left(g^{2^{m-2}}\right)^{2}$ respects this embedding of $K_{7}$, acts on its vertices with order 2, and fixes three of its vertices. Since this contradicts the lemma, we conclude that the orbit of each vertex of $K_{4 n+3}$ under $g$ must have length 1 or 2 .

If the action of $g$ has at least three orbits $\left\{v_{1}, v_{4}\right\},\left\{v_{2}, v_{5}\right\},\left\{v_{3}, v_{6}\right\}$ of length 2, consider the subgraph $G$ of $K_{4 n+3}$ consisting of these 6 vertices together with the edges $v_{1} v_{2}, v_{2} v_{3}, v_{3} v_{4}, v_{4} v_{5}, v_{5} v_{6}, v_{6} v_{1}$ that form a loop and the edges $v_{1} v_{4}, v_{2} v_{5}, v_{3} v_{6}$ that are the diameters of this loop. Then $G$ is invariant under $g$. As an abstract graph, however, $G$ is isomorphic to $M_{3}$, the Möbius ladder with 3 rungs, and the loop $v_{1} v_{2} v_{3} v_{4} v_{5} v_{6}$ is also invariant under $g$. In [F] it was proved that for any embedding of $M_{3}$ there is no such orientation reversing homeomorphism of $S^{3}$ leaving both $M_{3}$ and this loop invariant.

If $g$ has only 1 or 2 orbits of length 2 , the subgraph of $K_{4 n+3}$ spanned by the union of these orbits and of 5 or 3 additional vertices (necessarily fixed by $g$ ) provides an embedding of $K_{7}$, which contradicts the lemma.

Lastly, if $g$ has no orbit of length 2, it fixes all vertices and edges of $K_{4 n+3}$ pointwise. Then $g$ fixes an embedding of the Möbius ladder $M_{3}$ as a subgraph of $K_{4 n+3}$, which again contradicts [F], since $g$ is orientation reversing. Hence $K_{4 n+3}$ is intrinsically chiral.

\section{REFERENCES}

[CG] J. H. Conway and C. McA. Gordon, Knots and links in spatial graphs, J. Graph Theory 7 (1983), 445-453.

[F] E. Flapan, Symmetries of Möbius ladders, Math. Ann. 283 (1989), 271-283.

Department of Mathematics, Pomona College, Claremont, California 91711

Department of Mathematics, University of California, Berkeley, California 94720 